\title{
Actividad antifúngica de antioxidantes derivados de cuatro cultivares de Capsicum spp. contra hongos fitopatógenos
}

\section{Antifungal activity of antioxidants derived from four cultivars of Capsicum spp. against phytopathogen fungi}

\author{
Nancy Raquel López-Muñoz ${ }^{1}$ (), Mirella Romero-Bastidas ${ }^{1 *}$, Pablo Misael Arce-Amézquita ${ }^{1}$, José Saúl \\ Hernández-Rubio ${ }^{1}$ \\ ${ }^{1}$ Universidad Autónoma de Baja California Sur, Carretera al Sur KM 5.5, Colonia el Mezquitito, CP. 23080, La Paz, Baja California Sur, \\ México. \\ *Autor de correspondencia: miromero@uabcs.mx.
}

Artículo científico recibido: 08 de marzo de 2019 aceptado: 20 de junio de 2019

RESUMEN. El cultivo de chile, además del uso culinario posee actividad antimicrobiana, debido a la acción de antioxidantes. Por lo que es de interés en la producción agrícola, como alternativa en el manejo de enfermedades en plantas. El objetivo del estudio fue evaluar la capacidad antioxidante de cuatro variedades de chile y su efecto antifúngico contra Fusarium andiyazi y Cochliobollus spp., ambos son patógenos severos en el cultivo de espárrago y albahaca. A partir de muestras de frutos de chile habanero, jalapeño, serrano y morrón, se obtuvieron extractos etanólicos, donde se determinó su capacidad antioxidante. La acción antifúngica, se evaluó mediante dos técnicas; la prueba de zona de inhibición en PDA y la germinación de esporas en caldo de papa-dextrosa. La actividad de los extractos se evaluó mediante la medición del halo de inhibición, a los cinco días después de la siembra y la germinación de esporas a través de la cuantificación de las mismas a las $48 \mathrm{~h}$. Los resultados indican que el extracto de chile habanero presentó mayor capacidad antioxidante (11.9 $\mathrm{mg} \mathrm{g}^{-1}$ ) y porcentaje de inhibición contra $F$. andiyazi y Cochliobollus spp., (55.61 y $\left.82.29 \%\right)$, al tener respuesta similar al fungicida. La acción de inhibición de los patógenos en el resto de los extractos, varió entre éstos independiente de su capacidad antioxidante, donde posiblemente actúen otros compuestos. El extracto de chile habanero es eficaz para controlar Cochliobollus spp. y Fusarium andiyazi, y puede ser una alternativa para el control de estos fitopatógenos en la agricultura orgánica.

Palabras clave: Control, extractos, fenoles, inhibición, microrganismos.

ABSTRACT. Chili crop, in addition to the culinary use has antimicrobial activity, due to the action of antioxidants. For what is of interest in agricultural production, as an alternative in the management of diseases in plants. The objective of this study was to evaluate the antioxidant capacity of four varieties of chili and its antifungal effect against Fusarium andiyazi and Cochliobollus spp., both main severe pathogens in the cultivation of asparagus and basil. From samples of habanero, jalapeño, serrano and morrón fruits chili, ethanolic extracts were obtained where their antioxidant level was determined. The antifungal action was evaluated by two techniques; the inhibition zone test in PDA and the germination of spores in potatodextrose broth. The activity of the extracts was evaluated by measuring inhibition halo at five days after sowing and spore germination through the quantification at $48 \mathrm{~h}$. The results indicate that habanero pepper extract showed higher antioxidant capacity (11.9 $\mathrm{mg} \mathrm{g}^{-1}$ ) and percentage of inhibition against $F$. andiyazi and Cochliobollus spp. (55.61 and $82.29 \%$ ), having a similar response to fungicide. The action of inhibition of the pathogens in the rest of the extracts varied among them independent of their antioxidant capacity, where other compounds possibly act. Habanero pepper extract is effective to control Cochliobollus spp. and Fusarium andiyazi, and can be an alternative for the control of these phytopathogens in organic agriculture.

Key words: Control, extracts, inhibition, phenols, microorganisms. 


\section{INTRODUCCIÓN}

El género Capsicum, además de proveer proteínas y minerales necesarias en la dieta humana, posee propiedades terapéuticas y antimicrobianas (Lu et al. 2017). Su efecto, está asociado con la acción de antioxidantes, como fenoles, flavonoides, capsaicinoides, carotenoides, vitaminas, saponinas y terpenoides (Tag et al. 2014, lqbal et al. 2015). La mayoría de los estudios referentes a estas sustancias, se han dirigido principalmente a problemas de salud humana y poco se ha observado en patógenos relacionados con plantas (Dorantes et al. 2000). Una de las principales problemáticas comunes en campo, es el daño causado por enfermedades fúngicas (Knogge 1996). Como las provocadas por los hongos Fusarium andiyazi y Cochliobollus spp., principales patógenos en el cultivo de espárrago y albahaca, respectivamente. Debido a su rápida diseminación y poseen amplio rango de hospederos, lo que ocasiona la aplicación indiscriminada de fungicidas sintéticos para su control (López y Hermann, 2004).

Algunos estudios reportan que las sustancias presentes en los extractos de Capsicum, son de especial interés en el ámbito agrícola, para el control de microorganismos fitopatógenos (Corato et al. 2010). Estudios recientes, han comprobado que además de la capsaicina, se destaca la acción antifúngica de los fenoles, uno de los antioxidantes de mayor concentración que interfiere en el metabolismo microbiano, al inhibir su crecimiento (Kabara y Marshall 2005, Bacon et al. 2016). La baja toxicidad de éstas sustancias y su poca persistencia en el ambiente, lo hacen un excelente compuesto para ser empleado en la agricultura orgánica (Mahlo et al. 2010) para la producción de alimentos vegetales, donde hay una demanda creciente de alimentos inocuos; además de que se pretende reducir la contaminación y disminuir la emergencia de nuevas especies resistentes a los agroquímicos (Corato et al. 2010). Debido a lo expuesto anteriormente, los compuestos bioactivos de los extractos de Capsicum, podrían ser eficaces en la inhibición de diversas enfermedades fúngicas presentes en plantas cultivadas. Por lo anterior, el objetivo fue evaluar la actividad antifungica de antioxidantes derivados del extracto de cuatro cultivares de Capsicum spp. en la inhibición de F. andiyazi y Cochliobollus spp.

\section{MATERIALES Y MÉTODOS}

\section{Material vegetal}

Se utilizaron muestras de frutos de chile frescos, maduros y firmes de las variedades: habanero (Capsicum chinense), serrano (Capsicum annuum var. Serrano), jalapeño (Capsicum annuum var. Jalapeño) y pimiento morrón (Capsicum annuum var. annuum) que se obtuvieron en mercados regionales de La Paz, Baja California Sur, México durante el mes de septiembre del 2018.

\section{Cepas fúngicas}

Las cepas de F. andiyazi (FA0017) y Cochliobollus spp. (CH0016), se obtuvieron de la colección fúngica del Laboratorio de Fitopatología de la Universidad Autónoma de Baja California Sur, La Paz, México. Aisladas previamente de cultivos orgánicos de espárrago y albahaca, respectivamente. Para el análisis in vitro, se reactivaron las cepas mediante la toma de una alícuota de cada suspensión, la cual se inoculó en medio de cultivo papa dextrosa agar (Difco) e incubaron a $28^{\circ} \mathrm{C}$ por siete días.

\section{Preparación de los extractos vegetales}

La obtención de los diferentes extractos, se realizó mediante la metodología propuesta por Alam et al. (2015) con modificaciones. Los frutos de chile se lavaron con agua destilada estéril para eliminar partículas adheridas. La placenta con las semillas se removió y el resto del fruto se cortó en trozos de $0.5 \mathrm{~cm}$. La deshidratación de las muestras de frutos de chile, se llevó a cabo mediante congelamiento por $48 \mathrm{~h}$ en un liofilizador (Freezone, series Labconco). Posteriormente, la materia seca se maceró hasta obtener un polvo fino. El extracto etanólico (EE), se obtuvo pesando $7 \mathrm{~g}$ de la muestra seca, la cual se colocó en tubos de ensayo de $100 \mathrm{~mL}$, a los que se les añadieron $70 \mathrm{~mL}$ del solvente etanol al $96 \%$ sin desnaturalizar. Inmediatamente después, cada 
muestra se colocó en un sonicador (Branson 5210RMT, Ultrasonic Cleaner) por $10 \mathrm{~min}$. El sobrenadante fue sujeto a tres subsecuentes extracciones, repitiendo los pasos anteriores. La mezcla final, se filtró con papel filtro Whatman No. 1 (Whatman International) y el solvente se evaporó en un rota-evaporador (Buchi B-480 Waterbath) a $40{ }^{\circ} \mathrm{C}$, hasta su secado total. Para luego almacenar el extracto crudo concentrado a $-4{ }^{\circ} \mathrm{C}$ hasta su uso.

\section{Determinación de la actividad antioxidante}

La capacidad antioxidante en frutos de chile, se determinó con la metodología propuesta por Yamaguchi et al. (1998), mediante el reactivo 1,1Difenil-2-picrilhidrazil (DPPH), como compuesto antioxidante estándar. La curva estándar se obtuvo a partir de diluciones seriadas de una solución stock de 200 ppm de Trolox (6-hydroxy, 2, 5, 7, 8tetramethylchroman-2-carboxylic acid). El reactivo de DPPH se preparó, pesando $8 \mathrm{mg}$ de reactivo DPPH, disolviendo en metanol y aforando a $100 \mathrm{ml}$. Posteriormente se cubrió de la luz. Después de mezclar la solución del antioxidante (100 $\mu \mathrm{L})$ con la solución de DPPH (1 ml), se dejó reposar por $30 \mathrm{~min}$ en oscuridad, antes de hacer la medición. Las lecturas de absorbancia se realizaron a una longitud de onda de $517 \mathrm{~nm}$ en un espectrofotómetro (GENESYS ${ }^{\mathrm{TM}} 10 \mathrm{~S}$ UV-Vis Spectrophotometer - Thermo Fisher Scientific). Las muestras de extracto de chile, se midieron de la misma manera que el Trolox. El contenido de antioxidantes totales de cada muestra se expresó como mg equivalentes de Trolox por $\mathrm{g}$ del extracto etanólico (EE) a través de la curva de calibración con Trolox (mg ET* $\mathrm{g}^{-1} \mathrm{EE}$ ) (Figura 1).

\section{Cuantificación del contenido total de fenoles}

El contenido de fenoles totales en frutos de chile, se determinó por el método de Folin-Ciocalteau (Sigma), propuesto por Alam et al. (2015), usando ácido gálico como compuesto fenólico estándar. La curva estándar se obtuvo a partir de diluciones seriadas de una solución stock de ácido gálico de 50 ppm. La mezcla de reacción consistió en mezclar $100 \mu \mathrm{L}$ de ácido gálico con $250 \mu \mathrm{L}$ con reactivo de
Folin-Ciocalteu 2N, dejando reposar por $8 \mathrm{~min}$. Posteriormente se agregaron $1250 \mu \mathrm{L}$ de $\mathrm{Na}_{2} \mathrm{CO}_{3}$ al $7.5 \%$, y se dejó reposar por $2 \mathrm{~h}$. Las lecturas de absorbancia se realizaron a una longitud de onda de $765 \mathrm{~nm}$ en un espectrofotómetro (GENESYS ${ }^{\mathrm{TM}} 10 \mathrm{~S}$ UV-Vis Spectrophotometer-Thermo Fisher Scientific). Las muestras de extracto chile se analizaron de la misma manera que el ácido gálico. El contenido de fenoles totales de las muestras, se expresó como mg equivalentes de ácido gálico por gramo del extracto a través de la curva de calibración con ácido gálico ( $\mathrm{mg}$ $\left.\mathrm{GAE}^{*} \mathrm{~g}^{-1} \mathrm{EE}\right)$ (Figura 1).

\section{Evaluación in vitro de la actividad antifúngica Preparación del inóculo}

Para la preparación del inoculo fúngico utilizado con los extractos, se colectaron las esporas de los hongos presentes en las placas con agar, después de siete días de crecimiento, como lo describe Broekaert et al. (1990). La concentración de suspensión de esporas se estimó con una cámara de conteo, donde se cuantificó el número de esporas por mililitro de cada patógeno a evaluar.

\section{Inhibición del crecimiento micelial con el método de difusión de pozo en agar}

La actividad antifúngica de los cuatro extractos de chile, sobre el crecimiento micelial de los patógenos a evaluar, se determinó mediante la técnica de pozo en agar, propuesta por Ríos et al. (1988), la cual consiste en inocular por difusión, para lo cual se tomaron $100 \mu \mathrm{L}$ de la suspensión de esporas previamente preparada a concentración de $1 \times 10^{7}$ esporas $\mathrm{mL}^{-1}$ de cada patógeno y se sembraron en medio de PDA. Posteriormente se realizaron cuatro perforaciones equidistantes de $5 \mathrm{~mm}$ de diámetro en cada una de las placas, donde se colocaron 50 $\mu \mathrm{L}$ de extracto de chile en concentraciones de 300 ppm, como control negativo se utilizaron pozos que contenían $50 \mu \mathrm{L}$ de agua destilada estéril, mientras que control positivo fue el fungicida comercial Captan (1\% N-trichloromethylthio-4-cyclohexano-1,2dicarboximida) a $0.2 \mathrm{mg} \mathrm{mL}^{-1}$. Las placas se incubaron a $28 \pm 2^{\circ} \mathrm{C}$ y después de cinco días, se 
A)

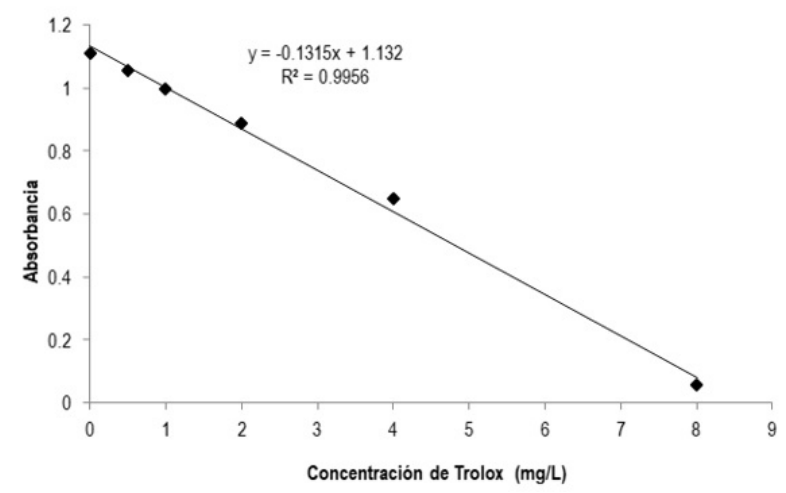

B)

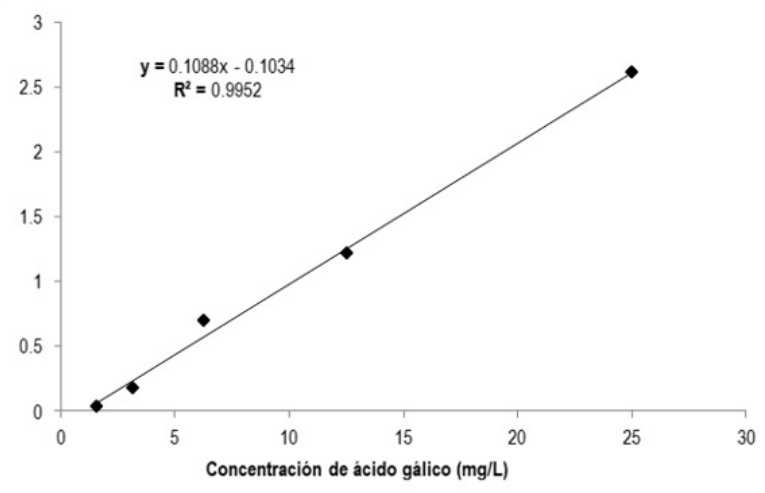

Figura 1. Curva estándar de inhibición de A) DPPH con trolox y contenido de fenoles (B).

determinó la actividad antifúngica de los extractos, al medir el diámetro de la zona de inhibición micelial en centímetros alrededor de los pozos.

\section{Efecto de los extractos en la germinación de es- poras}

El efecto de los extractos de chile, sobre la germinación de esporas, se realizó mediante la técnica del conteo de esporas germinadas en medio líquido, propuesta por Hadi et al. (2013). Para lo cual se tomaron $100 \mu \mathrm{l}$ de cada uno de los extractos, así como los tratamientos control, que se disolvieron en matraces que contenían $100 \mathrm{~mL}$ de medio líquido de papa-dextrosa. Posteriormente, un disco de $0.5 \mathrm{~cm}$ de diámetro con crecimiento activo de F. andiyazi o Cochliobollus spp. se colocó en matraces que se sellaron y agitaron en un Shaker (150 rpm) (Brinkmann Rockermix 1030). A las $48 \mathrm{~h}$ posteriores, se tomó una alícuota de $10 \mu \mathrm{l}$ de cada tratamiento y se cuantificó el número de esporas germinadas, mediante el uso del microscopio óptico (Labomed LX 400) y una cámara de conteo. La eficacia de los extractos, se expresó como porcentaje de inhibición de esporas germinadas, mediante la fórmula propuesta por Dissanayake (2014):

$$
\text { \% inhibición }=[(C-T) / C] X 100
$$

Dónde: C y T, representan las esporas germinadas del control $(\mathrm{C})$ y de los tratamientos $(\mathrm{T})$ con los ex- tractos, respectivamente.

\section{Diseño experimental}

Los resultados obtenidos de cada uno de los experimentos, se analizaron estadísticamente mediante un diseño completamente al azar, con tres repeticiones, realizándose análisis de varianza y comparación múltiple de medias de Tukey, mediante el paquete de diseños experimentales GraphPad Prism v.7.

\section{RESULTADOS}

\section{Propiedades antioxidantes de extractos de Cap-} sicum spp.

Para obtener una perspectiva completa del potencial antioxidante de los diferentes cultivares de chile habanero, serrano, jalapeño y morrón, se llevaron a cabo ensayos para evaluar la eliminación de radicales libre y el poder reductor total (Tabla 1). Todas las especies de chile, mostraron actividad antioxidante. Pero el extracto de chile serrano mostró el menor valor, al presentar $7.94 \mathrm{mg} \mathrm{g}^{-1}$ de antioxidantes, mientras que la mayor actividad lo presentó el extracto de chile habanero con $11.9 \mathrm{mg} \mathrm{g}^{-1}$, seguido del chile de morrón $\left(9.10 \mathrm{mg} \mathrm{g}^{-1}\right)$ y el chile jalapeño $\left(8.59 \mathrm{mg} \mathrm{g}^{-1}\right)$. Los resultados muestran que el extracto obtenido del pericarpio de chile habanero, fue el mejor en relación al contenido de antioxidantes. 
Tabla 1. Fenoles y actividad antioxidante de cuatro variedades de Capsicum spp.

\begin{tabular}{cccc}
\hline $\begin{array}{c}\text { Especie de } \\
\text { Capsicum }\end{array}$ & Tipo & $\begin{array}{c}\text { Actividad antioxidante } \\
\left(\mathrm{mg} \mathrm{g}^{-1}\right)\end{array}$ & $\begin{array}{c}\text { Fenoles Totales } \\
\left(\mathrm{mg} \mathrm{g}^{-1}\right)\end{array}$ \\
\hline C. chinense & Habanero & 11.9 & 43.63 \\
C. annuum & Serrano & 7.94 & 30.22 \\
C. annuum & Jalapeño & 8.59 & 46.76 \\
C. annuum & Morrón & 9.10 & 44.37 \\
\hline
\end{tabular}

\section{Contenido fenólico de los cuatro cultivares de Capsicum spp.}

El contenido de fenoles totales (CFT), se determinó por la prueba de Folin-Ciocalteu, de muestras liofilizadas del pericarpio de frutos de chile. El análisis del CFT, indica variaciones entre los extractos etanólicos (EE) de las muestras de Capsium (Tabla 1). El mayor contenido de fenoles totales se encontró en el extracto de chile jalapeño, con $46.76 \mathrm{mg} \mathrm{g}^{-1}$, mientras que en orden de importancia le siguió el pimiento morrón $\left(44.76 \mathrm{mg} / \mathrm{g}^{-1}\right)$ y habanero (43.63 $\left.\mathrm{mg} \mathrm{g}^{-1}\right)$; mientras que el serrano tuvo el menor contenido (30.22 $\mathrm{mg} \mathrm{g}^{-1}$ ).

\section{Actividad antifúngica de los extractos}

El análisis de varianza indica que los cuatro extractos etanólicos de Capsicum, tienen potencial antifúngico contra los hongos patógenos evaluados. Pero su efectividad en la inhibición del crecimiento micelial mostró diferencias significativas $(p<0.05)$ entre los extractos (Figura 2 y 3 ), ya que solo uno tuvo una marcada inhibición fúngica. Para el chile habanero se observó mayor inhibición de $F$. andiyazi y Cochliobollus spp., con halo de inhibición de 1.78 y $1.96 \mathrm{~cm}$. La respuesta expresada en el tratamiento de chile habanero, no tuvo diferencias significativas con el tratamiento del fungicida comercial (1.96 y 2.55 $\mathrm{cm}$ ), lo que demuestra que la eficacia de este extracto vegetal fue igual al del producto sintético que comúnmente se utiliza en campo. Mientras que los extractos de chile jalapeño $(1.47$ y $1.66 \mathrm{~cm})$, serrano $(1.45$ y $1.85 \mathrm{~cm}$ ) y morrón $(1.34$ y $1.1 \mathrm{~cm})$, presentaron menor inhibición en ambos patógenos. Al comparar la respuesta de los dos microorganismos patógenos sobre el efecto de los extractos de Capsicum y el fungicida, se observó que Cochliobollus spp. fue más susceptible a este tipo de sustancias, al mostrar un mayor diámetro de la zona de inhibición comparado con la respuesta de $F$. andiyazi a los mismos compuestos (Figura 4).

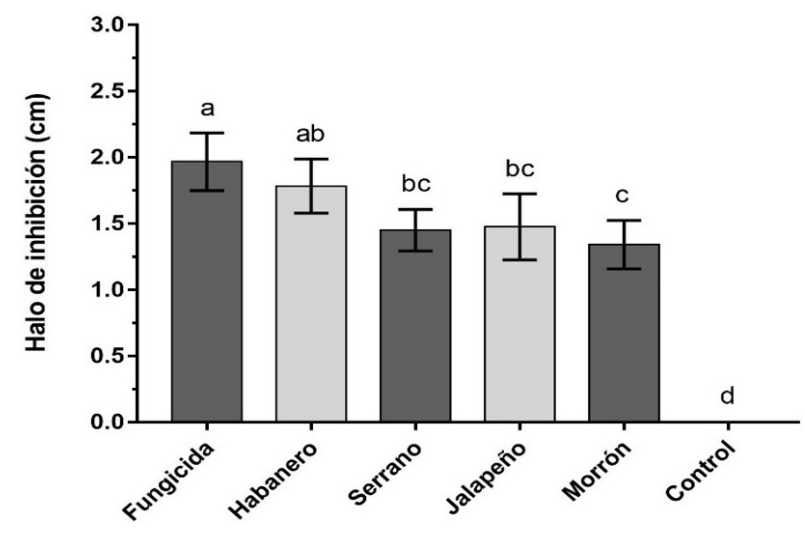

Figura 2. Extractos de Capsicum contra F. andiyazi.

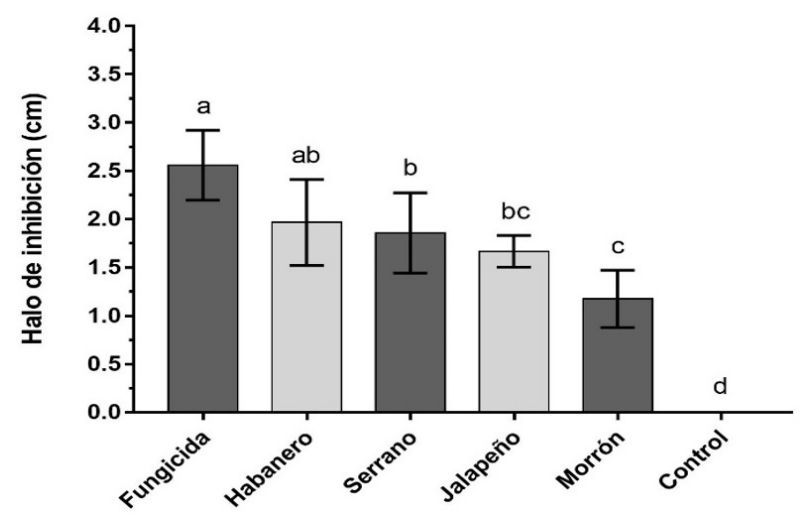

Figura 3. Extractos de Capsicum contra Cochliobollus spp.

\section{Inhibición de la germinación de esporas}

Entre los extractos de Capsicum evaluados, se observó que en el caso de $F$. andiyazi, los extractos de chile morrón, habanero y jalapeño, presen- 


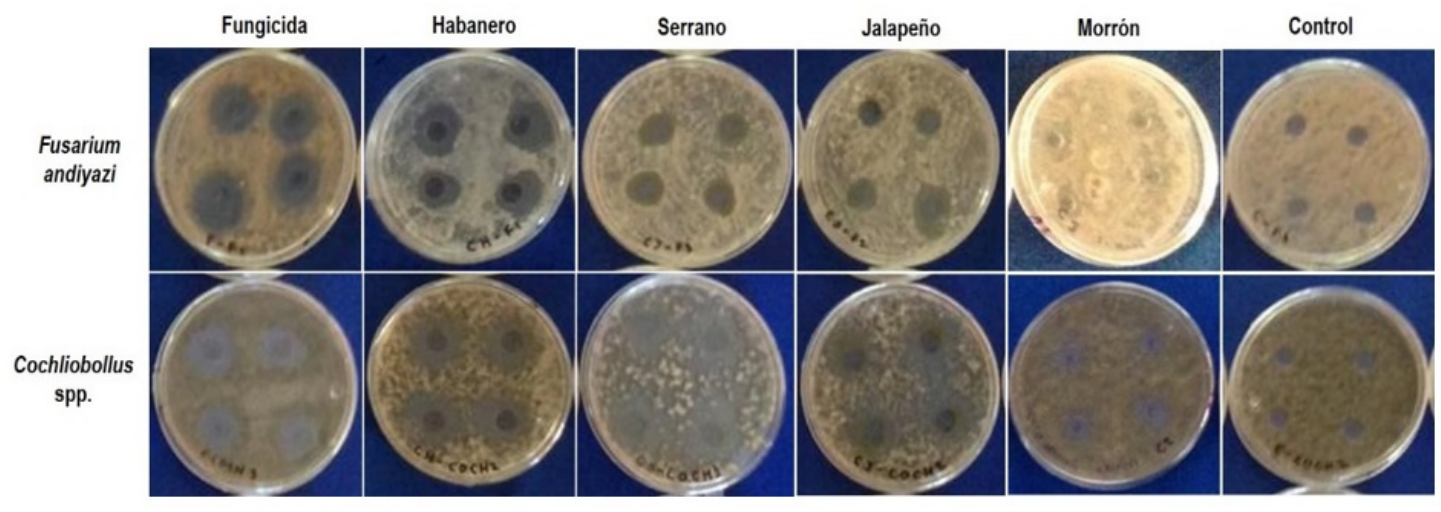

Figura 4. Bioensayo in vitro del efecto de extractos de Capsicum contra F. andiyazi y Cochliobollus spp.

taron mayor inhibición en la germinación de esporas al mostrar $64.87,55.61$ y $53.17 \%$, respectivamente. Éstos no presentaron diferencias significativas con respecto al tratamiento del fungicida $(74.63 \%)$, demostrando similitud en la efectividad antifúngica de dicho patógeno (Tabla 2). Mientras que, en el caso del extracto de chile serrano, éste solo presentó un porcentaje de inhibición de $9.75 \%$, siendo el que menor efecto tuvo contra la germinación de esporas de $F$. andiyazi.

Tabla 2. Efecto de extractos de Capsicum spp. en la inhibición de la germinación de esporas.

\begin{tabular}{lcc}
\hline \multicolumn{3}{c}{ Inhibición de la germinación de esporas (\%) } \\
\hline Tratamientos & Fusarium andiyazi & Cochliobollus spp. \\
\hline Habanero & $55.61 \mathrm{a}$ & $82.29 \mathrm{a}$ \\
Serrano & $9.75 \mathrm{~b}$ & $79.16 \mathrm{a}$ \\
Jalapeño & $53.17 \mathrm{a}$ & $44.79 \mathrm{~b}$ \\
Morrón & $64.87 \mathrm{a}$ & $72.91 \mathrm{ab}$ \\
Fungicida & $74.63 \mathrm{a}$ & $88.54 \mathrm{a}$ \\
\hline *Letras diferentes en las columnas indican diferencias signi- \\
ficativas $(\mathrm{p} \leq 0.05)$.
\end{tabular}

El efecto de los extractos de Capsicum en la germinación de esporas del hongo Cochliobollus spp. fue variable. Los resultados mostraron que el extracto de chile habanero $(82.29 \%)$ y serrano $(79.16 \%)$ fueron iguales estadísticamente junto con el fungicida comercial $(88.54 \%)$, al presentar mayor efecto de inhibición sobre el hongo. Sin embargo, estos tratamientos mostraron diferencias significativas con los extractos de chile morrón (72.91\%) y jalapeño (44.79). Al comparar el efecto de cada extracto, entre el crecimiento micelial y la germinación de esporas de F. andiyazi y Cochliobollus spp. se observó que el mayor efecto antifungico, se presentó sobre el hongo Cochliobollus spp. al mostrar más susceptibilidad a estos tipos de compuestos. No así en el caso de $F$. andiyazi, debido probablemente a que este último, es uno de los géneros más comunes en las plantas y el que está mayormente expuesto a diversas sustancias naturales o sintéticas, lo que le confiere algún tipo de tolerancia.

\section{DISCUSIÓN}

Diversos estudios indican que el chile habanero, es uno de los del genero de Capsicum que presenta mayor cantidad de compuestos antioxidantes, por ello, su consumo en diferentes tipos de presentaciones, es de gran importancia (Gurnani et al. 2016). Lo que se corroboró, ya que dentro de los diferentes extractos de Capsicum evaluados, el extracto de chile habanero presentó la mayor concentración de antioxidantes. Resultados similares fueron reportados por Castro-Concha et al. (2014), al evaluar por separado la capacidad antioxidante del pericarpio y la placenta de dos variedades de chile habanero (naranja y rojo) y encontraron que el chile habanero color naranja presentó mayor actividad antioxidante en el pericarpio y la placenta, pero este último tejido, superó cinco veces la capacidad antioxidante que el pericarpio. Esta respuesta podría deberse a que en la placenta se encuentran adheri- 
das las semillas, las cuales poseen la mayor concentración de fitoquímicos, como carotenoides, vitaminas, compuestos fenólicos y capsaicinoides (CastroConcha et al. 2012). También se observó que algunos extractos con menor contenido de fenoles, tuvieron menor capacidad antioxidante, como el caso del chile serrano, que presentó la menor cantidad de compuestos en el pericarpio y la placenta. En tanto en el resto de los extractos, dicha relación no fue concisa, ya que, en el caso del chile habanero, la actividad antioxidante fue mayor, pero no el contenido de fenoles. Este tipo de respuestas, podría estar relacionada a que no todas las plantas presentan una relación en la misma cantidad de estos compuestos, y a la vez pueden no presentar la misma función biológica durante los procesos relacionados a mecanismos fisiológicos de cada especie como lo menciona Gambacorta et al. (2009), al reportar que algunos tipos de fenoles, presentan acción protectora contra algunas enfermedades, mientras que otros contribuyen a dar forma, textura y color en frutos y plantas. Al respecto Gougoulias et al. (2012), mencionan que en el caso de los compuestos fenólicos, son considerados metabolitos secundarios que las plantas sintetizan directamente para adaptarse frente a condiciones de estrés. Sobre lo mismo NúñezRamírez et al. (2011), mencionan que las técnicas y los sistemas de cultivo, así como la fertilización, riego y variedad afectan los niveles de la actividad antioxidante en el cultivo de chile.

Estos resultados concuerdan con los de Bertão et al. (2016), quienes determinaron relación entre el contenido de compuestos bioactivos de diferentes genotipos de Capsicum (C. annuum L., C. baccatum L., C. chinense Jacq., $C$. frutescens L. y $C$. praetermissum) y su actividad antioxidante, encontrando que no todos tienen relación. Por el contrario Lee et al. (1995), observaron correlación lineal entre la concentración de fenoles y la actividad antioxidante en chiles, principalmente de tipo habanero. Para el contenido de fenoles, se observó una concentración variable en cada extracto de Capsicum spp., presentando el chile serrano la menor concentración. Lo que difiere de lo reportado por Cerón et al. (2016) quienes señalan que el chile serrano tuvo mayor con- tenido fenólico (144 mg $100 \mathrm{~g}^{-1} \mathrm{GAE}$ ) que el chile habanero (34 mg $100 \mathrm{~g}^{-1} \mathrm{GAE}$ ). De forma contraria, en los resultados obtenidos en los tratamientos del extracto de chile morrón y jalapeño, se observó que éstos presentaron la mayor concentración de compuestos fenólicos. Esta diferencia de concentración, podría estar ligada al estado de madurez de los diferentes tipos de chiles, los cuales la mayoría normalmente se consumen inmaduros. Al respecto, Marinova et al. (2005) y Navarro et al. (2006), encontraron que las frutas en la etapa de maduración verde representan más concentración de fenoles en sus tejidos que en estado maduro. De forma contraria Conforti et al. (2007) estudiaron la actividad antioxidante de chiles verdes y rojos de $C$. annuum L. var. Acuminatum, y encontraron que la concentración de compuestos orgánicos incrementó conforme los chiles alcanzaron la madurez. Así mismo, otros autores han encontrado que la concentración de dichas sustancias depende del cultivar y no de su estado fisiológico (Howard et al. 2000). Estos resultados coinciden con los reportados por MedinaJuárez et al. (2012), los cuales evaluaron el contenido total de fenoles en chile anaheim, morrón, caribe, jalapeño y serrano y encontraron que el extracto de chile morrón presentó el mayor nivel. Los diferentes niveles de fenoles totales obtenidos, también pueden atribuirse a las diversas especies de Capsicum seleccionadas, así como el procedimiento y los tipos de estándares utilizados, tal como lo señala Vashisth et al. (2011), quienes indican que para algunas variedades de chile, la deshidratación por congelamiento incrementa la porosidad del tejido, lo que provoca mayor eficiencia de extracción de compuestos fenólicos. Esto concuerda con los estudios realizados por Materska (2014), quien determinó el impacto del proceso de enfriamiento en el contenido de fitoquimicos en la placenta y pericarpio de diferentes variedades de chile $(C$. annuum var. Red Knight, C. annum var. Shanghai, C. annuum var. Socrates y $C$. annuum var. Capel) y observó que el mayor CFT se presentó en el pericarpio. Resultados contrarios son reportados por Ornelas-Paz et al. (2010), los cuales analizaron el contenido de fenoles totales por el método de cocción 
en muestras de chile poblano, bell, chilaca, caribe, jalapeño, serrano, habanero y manzano, concluyendo que se incrementó de forma secuencial en el CFT. Al respecto Loizzo et al. (2015), confirman que esta variación de respuestas indica que no todas las especies de chile son sensibles al mismo tipo de procesamiento, lo que puede tener impacto en el contenido de compuestos bioactivos. Así mismo, los resultados mostraron que el contenido de fenoles no está relacionado con la pungencia de la variedad de chile en estudio, la cual aunque no se determinó, si se conoce el nivel de Capsaicinoides. Como lo confirma Aguiar et al. (2016), quienes no reportan correlación entre el total de capsaicinoides y el contenido de fenoles totales, los cuales varían con el estado de madurez del fruto y color.

Las propiedades antioxidantes, antiinflamatorias, antialergénicos, antibacteriales y antimutagenicos, varia con la especie de Capsicum. Lo que está relacionado con la expresión de compuestos como, caroteonides, capsaicinoides y vitaminas (Loizzo et al. 2015). Los extractos de Capsicum spp. mostraron una respuesta antifúngica diversa en el control de hongos fitopatógenos. La comparación de la inhibición del crecimiento micelial de los extractos de chile y su respectiva concentración de compuestos fenólicos y antioxidantes, mostró, que el chile habanero, tuvo efecto dependiente de la concentración de antioxidantes, pero en el resto de los extractos no se encontró esta relación. Esto podría deberse al estado de maduración avanzada que presentaban dichos frutos $y / 0$ a la diferencia de coloración de su pulpa o tejidos, donde se ha señalado previamente la influencia de estas características en el contenido de este tipo de sustancias. Lo que indica que la actividad antifúngica del extracto de chile habanero fue mayor, debido probablemente al incremento de la concentración de compuestos antioxidantes. Lo que concuerda con lo reportado con Pan y Wang (2010), quienes señalan que el incremento de compuestos antioxidantes se correlacionan con el incremento de la tolerancia a diversos tipos de estrés. Estudios previos, han establecido que el extracto de chile habanero contiene numerosos compuestos con actividad antioxidante incluyendo $\beta$-carotenos, alcaloides, fenoles y ácido ascórbico (Shahidi 2000). Este tipo de compuestos antioxidantes podrían estar implicados en la supresión del crecimiento fúngico al dañar su metabolismo celular, como lo menciona Govindappa et al. (2011), quienes reportaron la actividad antifungica de antioxidantes derivados de Crotalaria pallida Aiton contra diferentes hongos fitopatogenos, incluyendo a $A$. nidulans, A. flaviceps, F. solani, F. oxysporum, F. verticilloides, y $N$. oryzae, atribuyendo la inhibición positiva a la presencia de compuestos polifenoles, como alcaloides, flavonoides, taninos, esteroides, terpenoides, fenoles y saponinas. Sobre lo mismo Srinivasan y Sarada (2012), encontraron resultados similares al reportar la actividad antifungica de derivados de fenoles aislados de Psoralea corylifolia contra F. oxysporum, F. moniliforme y F. graminearum. Mientras que Gurnani et al. (2016), evaluaron la actividad antimicrobiana y potencial antioxidante de semillas de Capsicum frutescens, encontrando una asociación entre la extracción de compuestos antioxidantes y la eficaz inhibición de los microorganismos patógenos evaluados, como Pesudomaonas aeruginosa, Klebsilla pneumonae, Staphylococcus aureus and Candida albicans, C. krusei, Alternaria alternata y Aspergillus niger.

Para los fenoles, diversos estudios han confirmado que muchos compuestos fenólicos poseen acción antimicrobiana. Su principal función es como precursores para la formación de polímeros estructurales como la lignina y moléculas de señalización (Akila et al. 2011). Diversos reportes indican que estos compuestos alteran la funcionalidad de la membrana (Lanciotti et al. 2014). Al respecto CruzRodríguez (2017), asociaron el efecto de los compuestos fenólicos aislados del extracto de Crotalaria longirostrata con la efectiva inhibición del crecimiento micelial y esporulación de $F$. verticillioides y A. flavus, debido a la inactivación de enzimas celulares. Sobre lo mismo Guzman de Peña (1998), señalan que cuando la enzima decarboxilasa ornitina es inhibida, el micelio y la esporulación sexual y asexual se bloquean, lo cual induce estrés en la membrana celular y conduce a reacciones que suprimen las proteínas relacionadas con el crecimiento celular, el desarrollo 
de hifas y la germinación de esporas. Otros estudios indican que los compuestos fenólicos inhiben el crecimiento celular a través de la desestabilización de la homeostasis celular redox y/o el sistema antioxidación (Hernández-Ortega et al. 2011). De la misma manera, estudios realizados por Wilson et al. (1997), informaron que los compuestos derivados de aceites esenciales de las variedades de $C$. annum, $C$. chinense y $C$. frutescens presentan alta actividad antifúngica al inhibir un $99 \%$ la germinación de esporas de Botrytis cinerea. Así mismo Rodríguez et al. (2015), reportaron la eficacia de extractos fenólicos derivados de chile chiltepin sobre la inhibición de $F$. oxysporum y $A$. alternata, señalando que esta respuesta podría deberse a la formación de complejos con proteínas solubles y extracelulares, generando una disrupción de la pared celular de los hongos. Además de inhibir las rutas enzimáticas vitales, como, el sistema P450 dependiente de oxidasa, mediante el bloqueo de las enzimas hidrolasas esteroides (Treutter 2005).

\section{CONCLUSIONES}

El extracto de chile habanero, presento mayor concentración de antioxidantes y actividad antifungica sobre el crecimiento micelial y la inhibición de la germinación de esporas de $F$. andiyazi y Cochliobollus spp. Mientras que el mayor contenido de fenoles se presentó en el extracto de chile jalapeño, pero con menor actividad antifungica. Estos resultados sugieren que algunos extractos de Capsicum spp. son una buena fuente de compuestos antioxidantes para la obtención de sustancias con acción antifúngica y posiblemente contra otras enfermedades microbianas. Los resultados muestran la posibilidad de usar extractos derivados de Capsicum spp. para el desarrollo de métodos ecológicos en el control de hongos fitopatógenos.

\section{LITERATURA CITADA}

Aguiar AC, Pereira CJ, Fernandez BG, Teixeira GH, Martínez J (2016) Comparative study of capsaicinoid composition in Capsicum peppers grown in Brazil. International Journal of Food Properties 19: 1292-1302.

Akila R, Rajendran L, Harish S, Saveetha K, Raguchander T, Samiyappan R (2011) Combined application of botanical formulations and biocontrol agents for the management of Fusarium oxysporum $\mathrm{f}$. sp. cubense (Foc) causing Fusarium wilt in banana. Biological Control 57: 175-183.

Alam MA, Juraimi AS, Rafii MY, Hami AA, Aslani F, Alam MZ (2015) Effects of salinity and salinity-induced augmented bioactive compounds in purslane (Portulaca oleracea L.) for possible economical use. Food Chemistry 169: 439-447.

Bacon K, Boyer R, Denbow C, O'Keefe S, Neilson A, Williams RC (2016) Evaluation of different solvents to extract antibacterial compounds from Jalapeno peppers. Food Science \& Nutrition 5: 497-503.

Bertão MR, Moraes MC, Palmieri DA, Pereira SL, Gonçalves DRM (2016) Cytotoxicity, genotoxicity and antioxidant activity of extracts from Capsicum spp. Research Journal of Medicinal Plants 10: 265-275.

Broekaert WF, Terras FRG, Cammue BPA, Vanderleyden J (1990) An automated quantitative assay for fungal growth inhibition. Federation of European Microbiological Societies 69: 55-60.

Castro-Concha LA, Canche-Chuc I, Miranda-Ham MDL (2012) Determination of antioxidants in fruit tissues from three accessions of habanero pepper (Capsicum chinense Jacq.). Journal of the Mexican Chemical Society 56: 15-18.

Castro-Concha L, Tuyub-Che J., Moo-Mukul A, Vazquez-Flota F, Miranda-Ham M (2014) Antioxidant capacity and total phenolic content in fruit tissues accessions of Capsicum chinense jacq. (Habanero pepper) at different stages of ripening. The Scientific World Journal. Article ID 809073. Doi: 10.1155/2014/809073. 
Cerón CTG, Santiesteban-López NA, Acle MR (2016) Combinación de dos extractos de Capsicum: composición y actividad antimicrobiana. Revista Iberoamericana de las Ciencias Biológicas y Agropecuarias 5: 1-19.

Conforti F, Statti GA, Menichini F (2007) Chemical and biological variability of hot pepper fruits (Capsicum annuum var. acuminatum L.) in relation to maturity stage. Food Chemistry 102: 1096-1104.

Corato U, Maccioni O, Trupo M, Di Sanzo G (2010) Use of essential oil of Laurus nobilis obtained by means of a supercritical carbon dioxide technique against post harvest spoilage fungi. Crop Protection 29: 142-147.

Cruz-Rodríguez RI, Meza-Gordillo R, Rodríguez-Mendiola MA, Arias-Castro C, Mancilla-Margalli NA, ÁvilaMiranda ME, et al. (2017) Antifungal activity of Crotalaria longirostrata Hook. \& Arn. extracts against phytopathogen fungi from maize. Gayana botanica 74: 167-175.

Dissanayake MLMC (2014) Inhibitory effect of selected medicinal plant extracts on phytopathogenic fungus Fusarium oxysporum. Annual Research \& Review in Biology 4: 133-142.

Dorantes L, Colmenero R, Hernández H, Mota L, Jaramillo ME, Fernández E, et al. (2000) Inhibition of growth of some food-borne pathogenic bacteria by Capsicum annum extracts. International Journal of Food Microbiology 57: 125-128.

Gambacorta G, Baiano A, Previtali MA, Terracone C, La Notte E (2009) Role of antioxidant substances in foods. Italian Journal of Agronomy 4: 171-184.

Govindappa M, Bharath N, Shruthi HB, Sadananda TS, Sharanappa P (2011) Antimicrobial, antioxidant and in vitro anti-inflammatory activity and phytochemical screening of Crotalaria pallida Aiton. African Journal of Pharmacy and Pharmacology 5: 2359-2371.

Gougoulias N (2012) Comparative study on the polyphenol content and antioxidant activity of some medicinal plants. Oxidation Communications 35: 1001-1010.

Gurnani N, Gupta M, Mehta D, Kumar MB (2016) Chemical composition, total phenolic and flavonoid contends, and in vitro antimicrobial and antioxidant activities of crude extract from red chilli seeds (Capsicum frutescens L.). Journal of Taibah University for Science 10: 462-470.

Guzmán de Peña D, Aguirre J, Ruiz HJ (1998) Correlation between the regulation of sterigmatocystin biosynthesis and asexual and sexual sporulation in Emericella nidulans. Antonnie Van Leeuwenhoek 73: 199-205.

Hadi M, Kashefi B, Sobhanipur A, Rezaarabsorkhi (2013) Study on effect of some medicinal plant extracts on growth and spore germination of Fusarium oxysporum schlecht. In vitro. American-Eurasian Journal of Agricultural and Environmental Science 13: 581-588.

Hernández-Ortega A, Luca F, Ferreira P, Medina M, Guallar V (2011) Modulating $\mathrm{O}_{2}$ reactivity in a fungal flavoenzyme: involvement of aryl-alcohol oxidase phe-501 contiguous to catalytic histidine. Journal of Biological Chemistry 286: 41105-41114.

Howard LR, Talcott ST, Brenes CH, Villalon B (2000) Changes in phytochemical and antioxidant activity of selected pepper cultivars (Capsicum species) as influenced by maturity. Journal of Agricultural of Food Chemistry 48: 1713-1720.

lqbal E, Salim KA, Lim LBL (2015) Phytochemical screening, total phenolics and antioxidant activities of bark and leaf extracts of Goniothalamus velutinus (Airy Shaw) from Brunei Darussalam. Journal of King Saud University-Science 27: 224-232.

Kabara JJ, Marshall (2005) Medium-chain fatty acids and esters. In: Davidson PM, Sofos JN, Branen AL (eds.) Antimicrobials in food. Marcel Dekker. New York. pp: 327-360. 
Knogge W (1996) Fungal infection of plants. The Plant Cell 8: 1711-1722.

Lanciotti R, Gianotti A, Patrignani F, Belletti N, Guerzoni ME, Gardini F (2014) Use of natural aroma compounds to improve shelflife and safety of minimally processed fruits. Food Science \& Technology 15: 201-208.

Lee Y, Howard LR, Villalón B (1995) Flavonoids and antioxidant activity of fresh pepper (Capsicum annuum) cultivars. Journal of Food Science 60: 473-476.

Loizzo MR, Pugliese A, Bonesi M, Menichini F, Tundis R (2015) Evaluation of chemical profile and antioxidant activity of twenty cultivars from Capsicum annum, Capsicum baccatum, Capsicum chacoense and Capsicum chinense: A comparison between fresh and processed peppers. LWT-Food Science and Technology 64: 623-631.

López G, Hermann M (2004) El cultivo del ulluco en la sierra central del Perú. CIP. Lima, Perú. 51p.

Lu A, Onoja U, Onyeke CC, Nweze El (2017) Antimicrobial activities of four varieties of Capsicum annuum fruits cultivated in Southeast Nigeria against multidrug-resistant and susceptible organisms. Journal of Basic Pharmacology and Toxicology 1: 21-26.

Mahlo SM, McGaw LJ, Eloff JN (2010) Antifungal activity of leaf extracts from South African trees against plant pathogens, Crop Protection 29: 1529-1533.

Marinova DF, Ribarova, Atanassova M (2005) Total phenolics and total flavonoids in Bulgarian fruits and vegetables. Journal of the University of Chemical Technology and Metallurgy 40: 255-260.

Materska M (2014) Bioactive phenolics of fresh and freeze-dried sweet and semi-spicy pepper fruits (Capsicum annuum L.). Journal of Functional Foods 7: 269-277.

Medina-Juárez LA, Molina-Quijada DMA, Del Toro SCL, González-Aguilar GA, Gámez-Meza N (2012) Antioxidant activity of peppers (Capsicum annuum $\mathrm{L}$ ) extracts and characterization of their phenolic constituents. Interciencia 37: 588-593.

Navarro JM, Flores P, Garrido C, Martinez V (2006) Changes in the contents of antioxidant compounds in pepper fruits at different ripening stages, as affected by salinity. Food Chemistry 96: 66-73.

Núñez-Ramírez F, González-Mendoza D, Grimaldo-Juárez O, Díaz LC (2011) Nitrogen fertilization effect on antioxidants compounds in fruits of habanero chili pepper (Capsicum chinense). International Journal of Agriculture and Biology 13: 827-830.

Ornelas-Paz JJ, Martínez BJM, Ruíz CS, Santana RV, Ibarra JV, Olivas GI, Pérez MJD (2010) Effect of cooking on the capsaicinoids and phenolics content of Mexican peppers. Food Chemistry 119: 1619-1625.

Pang $\mathrm{CH}$, Wang BS (2010) Role of ascorbate peroxidase and glutathione reductase in ascorbate-glutathione cycle and stress tolerance in plants. In: Anjum NA, Umar S, Chan MT (ed). Ascorbate-glutathione pathway and stress tolerance in plants. Springer Science. Berlin, Alemania. pp: 91-113.

Ríos J, Recio M, Villar A (1988) Screening methods for natural products with antimicrobial activity. Journal of Ethnopharmacology 23: 127-149.

Rodríguez MA, Troncoso RR, Sánchez EA, González MD, Ruiz SE, Zamora BR, et al. (2015) Efecto antifúngico de extractos fenólicos y de carotenoides de chiltepín (Capsicum annum var. glabriusculum) en Alternaria alternata y Fusarium oxysporum. Revista Argentina de Microbiología 47: 72-77.

Shahidi F (2000) Antioxidant factors in plant foods and selected oil seeds. BioFactors 13: 179-185. 
Srinivasan S, Sarada DVL (2012) Antifungal activity of phenyl derivative of pyranocoumarin from Psoralea corylifolia L. seeds by inhibition of acetylation activity of trichothecene 3-O-acetyltransfarase (Tri101). Journal of Biomedicine and Biotechnology 2012: 1-8.

Tag HM, Kelany OE, Tantawy HM, Fahmy AA (2014) Potential anti-inflammatory effect of lemon and hot pepper extracts on adjuvant-induced arthritis in mice. Journal of Basic and Applied Zoology 67: 149-157.

Treutter D (2005) Significance of flavonoids in plant resistance and enhancement of their biosíntesis. Journal of Plant Biology 7: 581-591.

Vashisth T, Singh RK, Pegg RB (2011) Effects of drying on the phenolics content and antioxidant activity of Muscadine pomace. LWT - Food in Science \& Technology 44: 1649-1657.

Wilson C, Solar J, El Ghaouth A, Wisniewski M (1997) Rapid Evaluation of Plant Extract and Essential Oils for Antifungal Activity Against Botrytis cinérea. Plant Disease 81: 204-210.

Yamaguchi T, Takamura H, Matoba T, Terao J (1998) HPLC method for evaluation of the free radical-scavenging activity of foods by using 1,1-Diphenyl-2-picrylhydrazyl. Bioscience, Biotechnology and Biochemistry 62: 1201-1204. 